\title{
Transfusion-Related Acute Lung Injury (TRALI) Following Urological Surgery-Alarming Reaction: A Case Report
}

Bhat $\mathbf{S}^{1}$, Kaul $\mathbf{A}^{2 *}$ and Karoli $\mathbf{R}^{3}$

${ }^{1}$ Department of Surgery Era Medical College, Lucknow, India

${ }^{2}$ Department of Nephrology Sanjay Gandhi Post Graduate Institute of Medical Sciences, Lucknow, India

${ }^{3}$ Department of Medicine, Era Medical College, Lucknow, India

\begin{abstract}
TRALI (Transfusion related acute lung injury) is characterized by rapid onset of respiratory distress following transfusion of blood products. We report a case of bilateral stone disease with hydronephrosis and hematuria. After surgery, following 1 hour of $100 \mathrm{ml}$ of blood transfusion, she developed severe respiratory distress with oxygen saturation $68 \%$ and sudden hypotension. The blood transfusion was stopped immediately and she was managed with mechanical ventilation that resulted in a gradual improvement of dyspnoea and oxygen saturation. There is wide discrepancy in literature with reported frequency as low as $1 / 557,000$ RBC units and as high as $1 / 432$ platelet units. The severity of symptoms can range from mild to severe, and the treatment requires oxygen support and mechanical ventilation. If treated properly, most patients with TRALI recover within 96 hours.
\end{abstract}

Keywords: Transfusion-related acute lung injury; Surgery

\section{Introduction}

Transfusion-related acute lung injury (TRALI) is one of the most common causes of transfusion associated major morbidity and death. It accounts for $13 \%$ of cases of transfusion-associated deaths [1]. Despite being the third leading cause of transfusion-related death, it is usually underdiagnosed and under reported [2]. It is defined as acute lung injury that occurs after transfusion of all types of blood products including whole blood, platelets, fresh frozen plasma, cryoprecipate, granulocytes, stem cell products and even IV immunoglobulin preparations [3-7] occurring within the first six hours following a transfusion [3]. There are some case reports with even packed red blood cell since there is some residual plasma in the packed cells also. Clinically and pathologically this condition is characterized by the sudden onset ofrespiratory distress, hypoxia, hypovolemia, hypotension, noncardiogenic pulmonary oedema, during or soon after blood transfusion occurring during or within a few hours of transfusion [4]. The aetiology has not yet been fully understood. Though most patients with TRALI who unlike the classical ARDS (Acute respiratory distress syndrome) have shorter course of illness and a lower mortality rate with majority showing recovery, awareness of the syndrome, its clinical diagnosis and temporal association to transfusion and supportive management which is complex and expensive, approaches for TRALI prevention must become evident.

\section{Case Report}

A 52-year-old female patient was admitted with the diagnosis of bilateral stone disease with bilateral hydronephrosis with hematuria. Bilateral Double-J stenting was done in this patient. In view of her anaemia, blood transfusion was planned for her. She received whole blood transfusion. Before blood transfusion, she was alert, oriented with no cardiorespiratory abnormality on clinico-radiological evaluation. Her vital parameters were normal and total leukocyte count was $8700 /$ $\mathrm{mm}$ [3] with serum creatinine of $2 \mathrm{mg} \%$.

After receiving approximately $100 \mathrm{ml}$ of blood for over 1 hour, she complained of sudden onset breathlessness, which progressively increased over the next 20 minutes. On examination she was febrile, tachypneic (respiratory rate $32 /$ minute), slightly cyanotic, with a pulse rate of $126 /$ minute and blood pressure $80 / 60 \mathrm{mmHg}$. Heroxygen saturation was $68 \%$ at room air. The jugular venous pressure (JVP) was normal and chest auscultation revealed bilateral basal fine inspiratory crackles with normal breath sounds. Sinus tachycardia was noted on an electrocardiogram (ECG). An urgent X-ray chest showed bilateral patchy infiltrates in the mid- and lower zones with normal heart size, suggestive of noncardiogenic pulmonary oedema. Echocardiography also ruled out cardiogenic dysfunction. Hematological investigations at this stage showed a total leukocyte count of $12300 / \mathrm{mm}$ [3], with no evidence of any haemolytic process. In the presence of $\mathrm{PaO}_{2} /$ $\mathrm{FiO}_{2}<300$ or $\mathrm{SPO}_{2} 90 \%$ on room air, Bilateral infiltrates on X-ray chest ECG showing no evidence of left atrial hypertension (i.e., circulatory over load), with no pre-existing Acute lung injury before transfusion and symptoms appearing within 6 hours of transfusion in absence of temporal relationship to any other alternative risk factor to acute lung injury a diagnosis of TRALI was made. The blood transfusion was stopped immediately and she was managed with mechanical ventilation that resulted in a gradual improvement of dyspnoea and oxygen saturation. She became completely asymptomatic after seven days and was discharged.

\section{Discussion}

TRALI is usually under- diagnosed and under- reported complication related to transfusion as many clinicians are not familiar with the syndrome. Characteristic clinical presentation of rapid development of non-cardiogenic pulmonary oedma within 6 hours of transfusion; no other risk factors for ALI except transfusion and when all other causes have been ruled out, a diagnosis of TRALI is made. The etiology of TRALI is currently not fully understood but is thought to be

*Corresponding author: Kaul A, Department of Nephrology Sanjay Gandhi Post Graduate Institute of Medical Sciences, Lucknow, India, Tel: 0522266 8700; Email: anupmaneph@gmail.com

Received June 05, 2014; Accepted October 29, 2014; Published November 20, 2014

Citation: Bhat S, Kaul A, Karoli R (2014) Transfusion-Related Acute Lung Injury (TRALI) Following Urological Surgery-Alarming Reaction: A Case Report. J Blood Disorders Transf 5: 236. doi: 10.4172/2155-9864.1000236

Copyright: ( 2014 Bhat S, et al. This is an open-access article distributed under the terms of the Creative Commons Attribution License, which permits unrestricted use, distribution, and reproduction in any medium, provided the original author and source are credited. 
immune mediated $[8,9]$. It has been observed that multiparous women who have higher rate of HLA sensitization with increasing number of pregnancies [8]. Transfusion of blood components obtained from these donors is thought to carry a higher risk of inducing immunemediated TRALI [9]. Previous transfusion or transplantation can also lead to donor sensitization. Thus plasma from multiparous women has been associated in causing impairment of pulmonary function in a randomized controlled trial [10]. To be at risk of TRALI, the blood recipient must express the specific HLA or neutrophil receptors to which the implicated donor has formed antibodies leading to release of cytokines and vasoactive substances that induce non-cardiac pulmonary edema. The antibodies are usually donor-derived, though there have been occasional reports of the syndrome occurring after transfusion of donor leucocytes which have interacted with either patient - derived antibodies or antibodies transfused in a second donation, or the presence of HLA antigen/antibody incompatibility in a pooled platelet concentrate.

TRALI is often a diagnosis of exclusion. The incidence of TRALI is increasing, possibly due to better recognition of its clinical presentation and temporal association to transfusion. The condition is characterized by the sudden onset of non-cardiogenic pulmonary oedema, often with marked systemic hypovolaemia and hypotension, occurring during or within a few hours of transfusion [4]. Fever and rigours have been observed in few cases but usually are relatively mild or absent. A clinical presentation of rapid onset respiratory distress with signs of pulmonary oedema presenting as pink frothy endotracheal secretions when all other causes of pulmonary oedema or ARDS have been ruled out, with radiological picture typically of ARDS in a setting of transfusion of blood products. Laboratory findings may show haemoconcentration and a sudden fall in serum albumin [11,12]. As in other causes of acute alveolar capillary leak, the pulmonary exudate in TRALI has high albumin content. Peripheral blood neutropenia has been reported but neutrophilia is more common $[13,14]$. As noted in our patient, is occasionally observed and is believed to be due to the sequestration of leukocytes in pulmonary circulation.

Since its clinical presentation as like pulmonary oedema which is not volume or cardiac related but due to altered vascular permeability in the lungs with exudation of fluid and protein into the alveoli, the conventional treatment of usage of diuretics and corticosteroids are not advocated but rather contraindicated .It is advised that ventilatory assistance and circulatory support which are the mainstays of treatment of TRALI should be provided.; maintenance of adequate circulating volume is the most beneficial and appropriate therapy, because the disease is self-limited, the majority of patients will respond to these therapies alone.

Prevention-It is largely impossible to prevent all cases of TRALI, but certainly a large group of such reactions can be prevented by following specific measures-evidence based practice for transfusion, increased use of optimal additive red cells containing less plasma is likely to reduce the clinical incidence and severity of the condition resulting from red cell transfusion. Women who have been immunized against leucocytes antigens during pregnancy account for the majority of implicated donors, thus donations from parous females may not be used for products containing large amounts of plasma, screening of donors for leucocytes antibodies needs active consideration and can stand as an important practical possibility in the future for regular donors of aphaeresis platelets or plasma.

\section{Conclusion}

Though most patients with TRALI who unlike the classical ARDS have shorter course of illness and a lower mortality rate with majority showing recovery, awareness of the syndrome, its clinical diagnosis and temporal association to transfusion and supportive management which is complex and expensive, approaches for TRALI prevention must become evident. It is essential for the clinicians to be aware of TRALI, where pulmonary oedema is seen during or after a transfusion they must differentiate between systemic fluids overload and ARDS. If TRALI is suspected, they should seek help from both in tensivists and haematologists for diagnosis and treatment. Thus, avoiding TRALI can be partly achieved by avoiding unnecessary transfusion, particularly of plasma and platelets and alternative usage of pooled plasma preparations rather than the standard single-donor FFP may prevent the condition.

\section{References}

1. Kopko PM, Marshall CS, MacKenzie MR, Holland PV, Popovsky MA (2002) Transfusion-related acute lung injury: report of a clinical look-back investigation. JAMA 287: 1968-1971.

2. Kleinman S, Caulfield T, Chan P Davenport R, McFarland J et al (2004) Toward an understanding of transfusion-related acute lung injury: statement of a consensus panel. Transfusion 44: 1774-1789.

3. Toy P, Popovsky MA, Abraham E, Ambruso DR, Holness LG, et al. (2005) Transfusion-related acute lung injury: definition and review. Crit Care Med 33 : 721-726.

4. Popovsky MA, Moore SB (1985) Diagnostic and pathogenetic considerations in transfusion-related acute lung injury. Transfusion 25: 573-577.

5. Rizk A, Gorson KC, Kenney L, Weinstein R (2001) Transfusion-related acute lung injury after the infusion of IVIG. Transfusion 41: 264-268.

6. Silliman CC, Paterson AJ, Dickey WO, Stroneck DF, Popovsky MA, et al. (1997) The association of biologically active lipids with the development of transfusionrelated acute lung injury: a retrospective study. Transfusion 37: 719-726.

7. Wallis JP, Lubenko A, Wells AW, Chapman CE (2003) Single hospital experience of TRALI. Transfusion 43: 1053-1059.

8. Dykes A, Smallwood D, Kotsimbos T, Street A (2000) Transfusion-related acute lung injury (Trali) in a patient with a single lung transplant. $\mathrm{Br} \mathrm{J}$ Haematol 109: 674-676.

9. Muller JY (2005) [TRALI: from diagnosis to prevention]. Transfus Clin Biol 12 95-102.

10. Palfi M, Berg S, Ernerudh J, Berlin G (2001) A randomized controlled tria oftransfusion-related acute lung injury: is plasma from multiparous blood donors dangerous? Transfusion 41: 317-322.

11. Dry SM, Bechard KM, Milford EL, Churchill WH, Benjamin RJ (1999) The pathology of transfusion-related acute lung injury. Am J Clin Pathol 112: 216221.

12. Wallis JP (2002) Transfusion related acute lung injury. Experience in a single institution. Vox Sang 83: 219.

13. Yomtovian R, Kline W, Press C (1984) Severe pulmonary hypersensitivity associated with passive transfusion of a neutrophil-specific antibody. Lance 1: $244-246$.

14. Van Buren NL, Stroncek DF, Clay ME, McCullough J, Dalmasso AP (1990) Transfusion-related acute lung injury caused by an NB2 granulocyte-specific antibody in a patient with thrombotic thrombocytopenic purpura. Transfusion 30: $42-45$

Citation: Bhat S, Kaul A, Karoli R (2014) Transfusion-Related Acute Lung Injury (TRALI) Following Urological Surgery-Alarming Reaction: A Case Report. J Blood Disorders Transf 5: 236. doi: 10.4172/2155-9864.1000236 